\title{
Active flow control by means of synthetic jets on a highly loaded compressor cascade
}

\author{
V Zander $^{1 *}$, M Hecklau ${ }^{1}$, W Nitsche ${ }^{1}$, A Huppertz $^{2}$, and M Swoboda ${ }^{2}$ \\ ${ }^{1}$ Department of Aeronautics and Astronautics, Technische Universität Berlin, Berlin, Germany \\ ${ }^{2}$ Rolls-Royce Deutschland Ltd \& Co. KG, Dahlewitz, Germany
}

The manuscript was received on 19 April 2011 and was accepted after revision for publication on 21 April 2011.

DOI: $10.1177 / 0957650911410275$

\begin{abstract}
This article presents the potential of active flow control to increase the aerodynamic performance of highly loaded turbomachinery compressor blades. Experimental investigations on a large-scale compressor cascade equipped with 30 synthetic jet actuators mounted to the sidewalls and the blades themselves have been carried out. Results for a variation of the inflow angle, the jet amplitude, and the actuation frequency are presented. The wake measurements show total pressure loss reductions of nearly 10 per cent for the synthetic jet actuation. An efficiency calculation reveals that the energy saved by actuation is nearly twice the energy consumption of the synthetic jets.
\end{abstract}

\section{INTRODUCTION}

Flow separation occurring on compressor blades of turbomachineries causes increasing performance losses and limits the working range of the aeroengine. Avoiding flow separation results in restricted stage pressure ratios, and thus in a large number of compressor stages. Increasing total pressure ratios of modern civil aero-engines have led to compressors with more than 15 stages [1]. Therefore, a lot of effort has been spent in the past years to increase the stage pressure ratio without flow separation. Reducing the number of stages will result in a lighter and cost-effective compressor compared to a turbomachine with the same overall pressure ratio. Especially, the methods of active flow control (AFC) are well suited to avoid flow separation in case of further stage pressure ration increases. Since the first AFC experiments realized by Prandtl, the AFC methods have been advanced and are used nowadays in a

*Corresponding author: Department of Aeronautics and Astronautics, Technische Universität Berlin, Marchstrasse 12, 10587 Berlin, Germany.

email: vincent.zander@ilr.tu-berlin.de variety of applications, even in the field of compressor aerodynamics. Many different control concepts have been proved in the meantime. However, the concept of boundary layer suction as proposed by Prandtl [2] to delay or suppress flow separation by removing the low-energy fluid is still used even in axial compressors [3]. The opposite is to reenergize the boundary layer by blowing compressed air out of slots or holes to avoid flow separation. In this case, it is important to distinguish between steady and unsteady flow control concepts. Steady concepts, as published for example by Nerger et al. [4], are an efficient method to improve compressor performance in case of overcritical design. As shown by Seifert et al. [5], the concept of steady blowing is highly energyconsuming compared to unsteady blowing. The required mass flow of compressed air can be strongly reduced with pulsed blowing [6]. This concept takes advantage of existing shear layer instabilities to avoid flow separation with a reduced flow control effort [5]. Spending less bleed air to control flow separation is a major goal when applying these AFC methods. The technical effort using boundary layer suction or blowing methods is immense due to the piping and control mechanism needed. Therefore, another flow 
control concept was introduced by Smith and Glezer [7]. They presented the synthetic jet actuator, which works without any external air supply. The ambient air is periodically sucked into the actuator and afterwards exhausted to the boundary layer. Due to this, the net mass flux for a complete actuator cycle is zero. This does not hold for the momentum that is generated by the actuator. Even these actuators have already been applied to axial compressors to reduce secondary flow effects, for example by Metejka et al. [8] and Zheng et al. [9]. Unlike in the present experiment, both authors apply the SJAs only to the casing. Furthermore, the SJAs have been asymmetrically distributed to the test sections, which limits the significance of the results. In the study presented here, 30 synthetic jet actuators were applied to a stator compressor cascade to reduce flow losses caused by secondary flows and separation. The actuators have been mounted symmetrically to the casing and the blade suction side. Detailed wake measurements have been performed to quantify the efficiency of the AFC approach. Measurements have been performed for different actuator configurations and cascade inflow conditions.

\section{EXPERIMENTAL SET-UP}

For the experimental results presented here the low-speed cascade test facility at the Department of Aeronautics and Astronautics at Technische Universität Berlin was used. Figure 1(a) shows a sketch of the cascade test section. As can be seen, the cascade consists of seven blades with additional tailbords at bottom and top. In front of the tailboards, a boundary layer suction is installed to obtain a pitchwise periodic flow in the test section. The suction rate is adjusted corresponding to the static pressure distribution measured in front of the blades. An inflow angle variation in the range of $\beta_{1}=55-65^{\circ}$ is feasible by rotating the disc the cascade is mounted to.
Changing the inflow angle requires an adaption of the bottom and top wall at the test section inlet as well. A sketch of the profile geometry is shown in Fig. 1(b). The corresponding parameters are summarized in Table 1. The design goal was a high-loaded blade profile with a flow turning as high as possible considering a representative pressure distribution. The $c_{p}$ distribution was scaled from high-subsonic flow conditions to incompressible flow conditions. This results in a flow turning of $\Delta \beta_{1}=60^{\circ}$. For the AFC experiments, a blade aspect ratio of $A R=0.8$ was chosen to enhance secondary flow effects. The blades have been scaled to allow an integration of the synthetic jet actuators inside of the blades. As suggested by the arrows in Fig. 1(a), all six cascade passages have been symmetrically equipped with SJAs. Six actuators have been mounted to both cascade sidewalls. Additionally, three actuators have been placed inside each blade to enable flow control in the rear part of the blade suction side (Fig. 2(b)). A total of 12 sidewall actuators and 18 blade surface actuators have been utilized for the experiments.

\section{SYNTHETIC JET ACTUATOR}

An SJA is similar in construction to a Helmholtz resonator. To generate a flow out of the orifice, the cavity volume is varied (Fig. 2(a)). A number of different SJA concepts can be found in the literature. They usually differ in the form of the driver that is needed to change the cavity volume for the synthetic jet generation. The actuators developed for the cascade experiments presented here use a piezodiaphragm to keep the system complexity as low as possible. Applying an AC voltage to the piezodiaphragm results in a periodic deflection of the membrane and thus in a cavity volume change. Matching the resonance frequencies of the piezo and the Helmholtz resonator results in high jet velocities. The maximum actuator exit velocity that was reached with the applied actuators
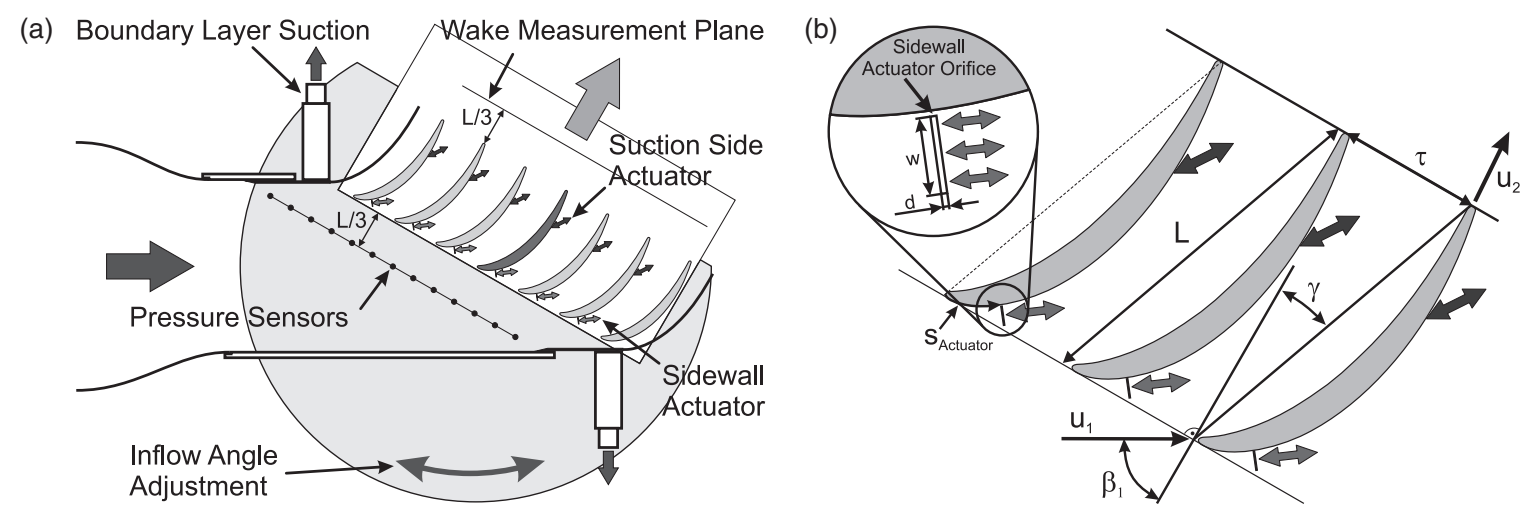

Fig. 1 Experimental set-up 
is $u_{j}=35 \mathrm{~m} / \mathrm{s}$ during the blowing cycle. This causes a flow separation at the actuator slot that forms a vortex ring, as illustrated in (Fig. 2(a)). This vortex moves away from the actuator orifice under its self-induced velocity. The vortex rings undergo transition to become fully turbulent and form a turbulent jet further downstream. A detailed investigation of the jet development is given by Glezer and Amitay [10]. The momentum generated during the blowing cycle can be used to reenergize the boundary layer. In the subsequent suction phase low-momentum fluid will be removed out of the boundary layer.

For the experimental investigations presented here two different types of SJAs have been developed since different slot geometries were used for the cascade sidewalls and the actuators mounted on the blade suction side. This requires a redesign of the SJA geometry to match the resonance frequencies of the piezo disc and the Helmholtz resonator. Moreover, both actuator types use different types of piezos. The sidewall actuators are driven by a rectangular piezo, whereas a circular piezo disc is used for the blade-mounted actuators to enable an integration of the SJAs into the blade suction side. During the actuator design process, different signal shapes were tested to drive the piezos. Maximum jet velocities were reached with a bipolar square wave signal

Table 1 Blade geometry data

\begin{tabular}{ll}
\hline Parameter & Value \\
\hline Chord length & $L=375 \mathrm{~mm}$ \\
Blade pitch & $\tau=150 \mathrm{~mm}$ \\
Blade height & $h=300 \mathrm{~mm}$ \\
Inflow angle & $\beta_{1}=55-65^{\circ}$ \\
Flow turning & $\Delta \beta=60^{\circ}$ \\
Stagger angle & $\gamma=20^{\circ}$ \\
Mach number & 0,07 \\
Reynolds number & 600000 \\
Inlet turbulence & $T u=1,25 \%$ \\
\hline
\end{tabular}


Fig. 2 Synthetic Jet Actuator for the cascade experiments due to the fast change of the piezo polarity and the associated high disc acceleration. A further advantage is the simple design of the piezo amplifier using square wave signals only. Therefore, maximum jet velocities of $u_{j}=25 \mathrm{~m} / \mathrm{s}$ for the sidewall-mounted actuators and $u_{j}=35 \mathrm{~m} / \mathrm{s}$ for the blade surface actuators were reached. To compensate for manufacturing tolerances that strongly influence the resonance frequencies of the SAJs, each actuator is driven by a separate signal generator and a high-voltage amplifier. Due to this, it is possible to synchronize the jet exit velocities of all actuators, to ensure that a homogenous flow control set-up can be realized. With a separate control unit, each actuator can be switched on individually. To drive all actuators in phase, amplitude modulation was used for the synthetic jet driving signal. Therefore, a second square wave signal was superimposed to all individual driving signals of the piezo discs. This enables a continuous frequency variation up to $F=240 \mathrm{~Hz}$ keeping the jet velocity constant. Figure 2(b) shows a picture of the actuator set-up.

\subsection{Sidewall actuator}

The sidewall actuators are mounted at $s / S=14.5$ per cent of the suction side length. For these actuators, rectangular slots are used which are perpendicularly oriented to the local blade contour (Fig. 1(b)). The actuator slot has a width of $w=20 \mathrm{~mm}$ and a depth of $d=0.3 \mathrm{~mm}$. For the sidewall actuators, a variation of the jet angle $\phi$ related to the sidewall has been carried out. Two different jet angles of $\phi=90^{\circ}$ and $\phi=35^{\circ}$ have been investigated during the windtunnel experiments. Here, only the results for $\phi=35^{\circ}$ are presented, because the wake measurements for $\phi=90^{\circ}$ show nearly no beneficial effects on the cascade losses, whereas remarkable loss reductions were achieved for the reduced jet angle. The experimental 
results for the jet angle variation are in good agreement with numerical results performed for this test configuration as presented by Gmelin et al. [11].

\subsection{Suction side actuator}

Preliminary oil flow visualization experiments reveal a highly three-dimensional (3D) flow field on the blade suction side. Figure 3 presents the results for $R e=600000$ and inflow angles $\beta_{1}=55^{\circ}$ on the left side and $\beta_{1}=60^{\circ}$ on the right, respectively. Additionally, the positions of the SJAs are shown. Strong corner vortices start developing at the position of the sidewall actuators which narrow the effective flow passage. In the rear part of the suction side, the flow is fully separated from the blade suction side. Therefore, three SJAs have been integrated to the blade suction side surface. It has been attempted to position the actuators along the separation line for an inflow angle of $\beta_{1}=60^{\circ}$. Due to constructive limitations, the centre actuator had to be moved downstream of the separation line. As for the sidewall actuators, rectangular slots have also been used for the blade actuators. The slot depth $d$ is the same as for the sidewall-mounted actuators. The slot width could be increased to $w=35 \mathrm{~mm}$. The centre actuator has been placed at $s / S=66$ per cent. The outer blade surface actuator slots have been shifted $y / h= \pm 15$ per cent in spanwise direction and were additionally moved upstream in axial direction to $s / S=59$ per cent. According to the shape of the separation

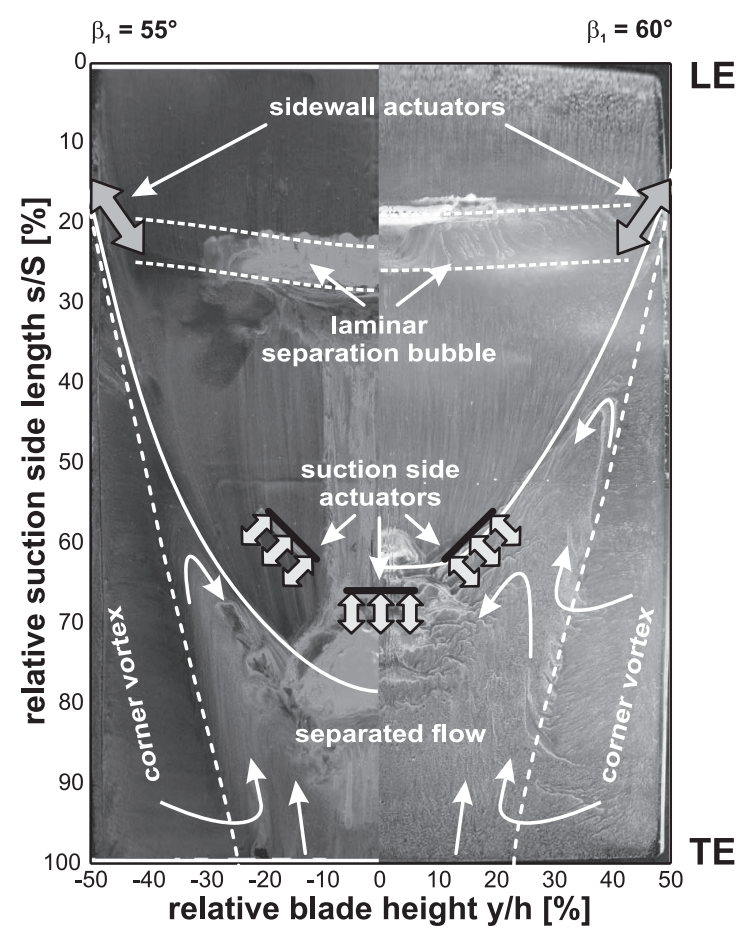

Fig. 3 Actuator configuration line, they have been rotated by $45^{\circ}$. The jet angle was fixed to $\phi=35^{\circ}$, because the results for the sidewall actuators reveal that small injection angles are more efficient.

The authors are aware that normal compressor blades are really thin, and that an integration of actuators is rarely possible. However, this technology can also be used for engine components that are placed inside the bypass duct and provide a sufficient thickness for integration.

\section{BASEFLOW RESULTS}

The oil flow visualization results for the base flow configuration have been briefly discussed in the previous chapter. The blade suction side presented in Fig. 3 shows a laminar flow region downstream of the leading edge (LE). For both inflow angles, transition occurs over a laminar separation bubble as can be clearly seen by the colour accumulation. Reducing the inflow angle to $\beta_{1}=55^{\circ}$ shifts the separation bubble further downstream and the streamwise length of the bubble shortens. In both cases, the sidewall actuators are placed upstream of the separation bubble. Oil flow visualization experiments have also been conducted in the case of AFC to ensure that the sidewall actuators did not trip the boundary layer to turbulent (not shown here). Together with the laminar separation bubble, the corner vortices between the suction side and the endwalls begin to develop. After the turbulent reattachment, the flow field develops a more and more 3D character. The area of attached flow decreases in axial direction due to the fast-growing corner vortices. For $\beta_{1}=60^{\circ}$ the flow fully separates at approximately 63 per cent of the suction side length. Reducing the blade loading results in a downstream shift of the separation to nearly 80 per cent. The corner vortex development is only slightly affected by the inflow angle variation. In both cases, the corner vortices cover nearly one quarter of the blade span at the trailing edge (TE).

An areal measurement for the pressure distribution has also been conducted in the case of no flow control. More details about this measurement can be found in reference [12]. Figure 4(a) shows only the pressure distribution at midspan. For the design inflow angle of $\beta_{1}=60^{\circ}$, a strong suction peak is seen at about $x / L=5$ per cent on the suction side. Afterwards, the pressure increases slightly. A pressure plateau between $x / L=14$ per cent and $x / L=21$ per cent caused by the separation bubble is not visible as expected. However, the turbulent reattachment downstream of $x / L=21$ per cent is clearly recognizable by the strong pressure rise. It seems that the surface pressure rises up to 
$x / L=75$ per cent. In fact, the flow is fully separated at $x / L=63$ per cent as seen in the oil flow visualization results. Unfortunately, no pressure taps have been placed in this region, due to the integration of the blade surface actuators. Nevertheless, a pressure plateau can be detected in the rear part of the blade suction side that corresponds to the flow separation. On the pressure side, rising $c_{p}$ values can be seen up to 10 per cent of the chord length. Afterwards, the pressure distribution shows no more significant changes down to the TE. Decreasing the inflow angle to $\beta_{1}=55^{\circ}$ reduces the blade loading and causes a downstream shift of the suction side pressure distribution as already observed in the oil flow visualization. The suction peak has shifted to $x / L=15$ per cent. This measurement also shows the pressure plateau of the laminar separation bubble. After the turbulent reattachment, the pressure rises continuously up to the blade TE. The overall pressure level of the suction side is lower compared to the design inflow angle. even the TE pressure is lower, which indicates higher pressure losses. The inflow angle reduction causes also great changes on the pressure side. A laminar flow separation can be detected at the LE. After reattaching to the surface, the pressure rises slowly and reaches a constant level in the second half of the profile chord. This differing flow topology can also be observed in the wake measurements that were performed with a five-hole probe one, third of the chord length downstream of the TE. The total pressure loss coefficient $\zeta_{q 1}$ was calculated according to the following expression

$$
\zeta_{q 1}=\frac{p_{t 1}-p_{t 2}}{q_{1}}
$$

wher $p_{t 1}$ and $p_{t 2}$ denote the total pressure at the inlet (1) and in the wake measurement plane (2) respectivly. The pressure difference is normalized with the dynamic pressure $q_{1}$ measured at the cascade inlet. The measurement accuracy for the total pressure is within 1 per cent and for the total pressure loss coefficient within 1,5 per cent. Figure 4(b) shows the total pressure loss coefficient for $\beta_{1}=55^{\circ}$ on the left and $\beta_{1}=60^{\circ}$ on the right, respectively. The loss coefficient is plotted only for a half blade span due to the symmetry of the flow field. Both measurements show maximum losses for the corner vortex located at $y /$ $h= \pm 30$ per cent blade span on the suction side. For the increased inflow angle, these high loss coefficients expand up to blade midspan, caused by the strong flow separation on the suction side. Minor losses can be seen in the vicinity of the pressure side. For $\beta_{1}=55^{\circ}$, weaker pressure losses are observed at midspan for the suction side, due to the delayed flow separation, whereas big differences can be seen for the pressure side. High loss coefficients can be observed for another vortex located on the pressure side. The appearance of this vortex is closely related to the laminar separation bubble at the LE of the pressure side and vanishes if the bubble length is reduced.

\section{AFC RESULTS}

Analyzsing the baseflow revealed strong secondary flow structures and large areas of separated flow. Therefore, the SJAs have been carefully placed on the cascade sidewalls and the suction side surface. Wake measurements have been performed in the same manner as for the baseflow to quantify the potential of the SJAs reducing the cascade losses. Pressure measurements have been carried out for midspan as well. A parameter study varying the inflow conditions and the actuator parameters has been conducted. First, the results for a jet amplitude variation will be presented. As mentioned in the actuator description, the maximum exit velocity of the (a)

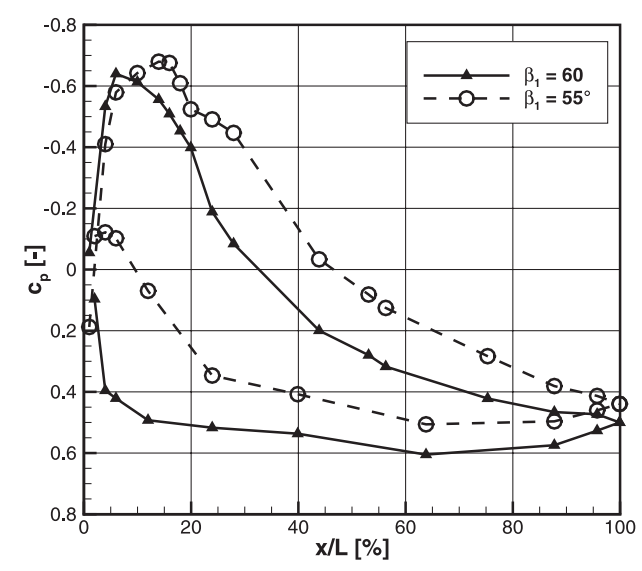

(b) $\quad \beta_{1}=55^{\circ}$

$\beta_{1}=60^{\circ}$

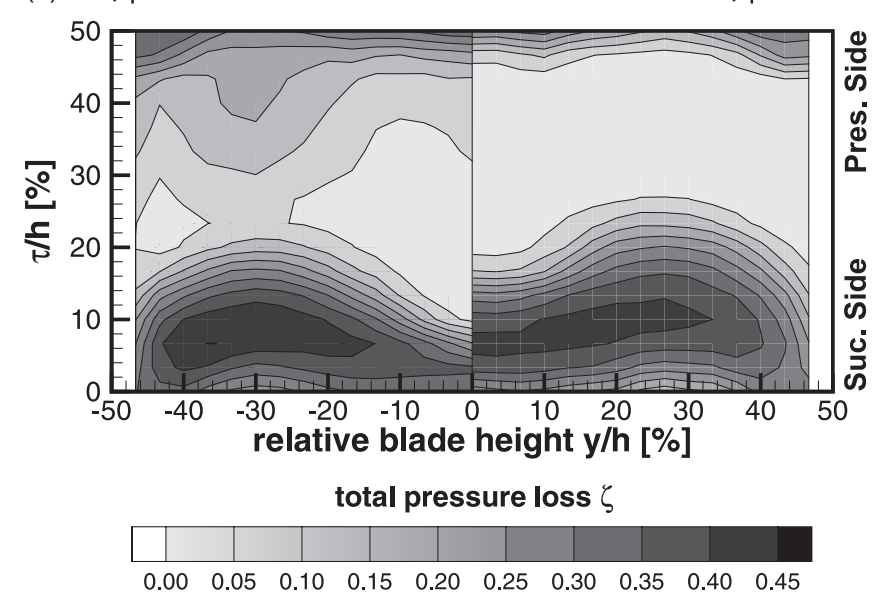

Fig. 4 Baseflow results 
sidewall actuators is limited. Due to this, the maximum jet velocity ration was limited to $u_{j} / u_{1}=1$, which is in the range of turbomachinery applications. Figure 5(a) shows the results for $\beta_{1}=60^{\circ}$ using the sidewall actuators in conjunction with the two outer blade surface-mounted synthetic jets. The amplitude modulation frequency was fixed at $F=230 \mathrm{~Hz}$. To enable a result comparison, the spanwise mass-averaged total pressure loss has been integrated over the blade span using the following formulation

$$
\zeta_{t}=\frac{2}{h} \int_{0}^{h / 2} \zeta_{q 1}(y) d y
$$

The integrated total pressure loss $\zeta_{t}$ is plotted in Fig. 5(a) for different jet velocity ratios between $u_{j}$ / $u_{1}=0.7$ and $u_{j} / u_{1}=1$. For reasons of comparison, the value for the baseflow is plotted at $u_{j} / u_{1}=0$ as well. With increasing jet velocity ratios a continuous decreasing pressure loss can be seen. A nearly linear correlation can be observed for the jet velocity ratios concerning the losses. Much higher jet velocities will result in even higher loss reductions as reported for example by Zheng et al. [9]. Velocity ratios much higher than one cannot be applied to realistic turbomachine conditions, because of the transonic inflow to the blade rows. Afterwards, a frequency variation was performed for the same inflow conditions. The jet velocity ratio was fixed to $u_{j} / u_{1}=1$. In Fig. 5(b) the integral total pressure loss value $\zeta_{t, A F C}$ is plotted over the amplitude modulation frequency $F$. The horizontal line at the top marks the value without synthetic jet actuation. It can be seen that the measured pressure losses depend only slightly on the amplitude modulation frequency. Minimum losses can be observed for the frequencies of $F=60 \mathrm{~Hz}$ and $F=230 \mathrm{~Hz}$. The corresponding dimensionless frequencies based on the suction side length and the

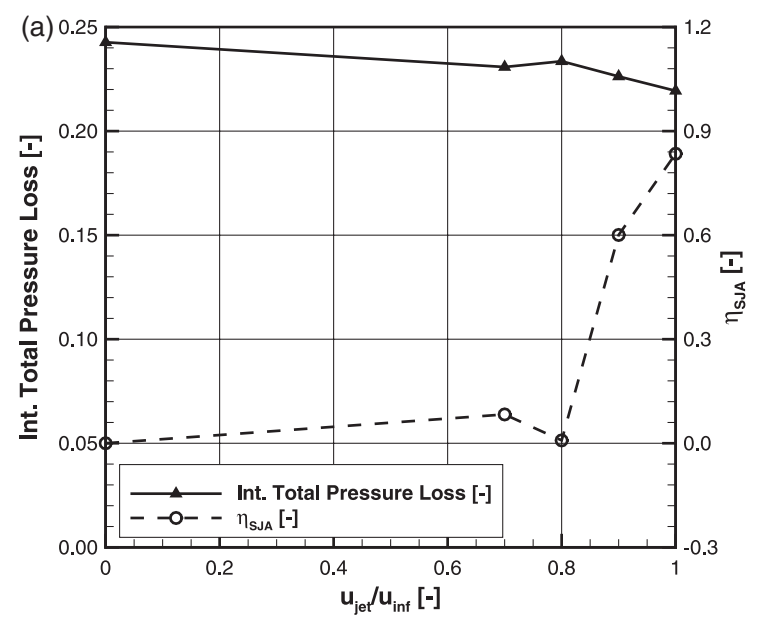

inflow velocity $F^{+}=f S / u_{1}$ are $F^{+}=1.03$ and $F^{+}=3.94$, respectively. The first value is close to one which is a common value for flow control applications. Nevertheless, actuation was most effective for $F^{+}=3.94$ in these measurements. Furthermore, the calculation of $\mathrm{F}^{+}$depends strongly on the scaling parameters. In Seifert et al. [5] it is suggested to use local quantities for the calculation. However, using different actuation positions at the same time makes a detection of useful scaling parameters difficult.

As a result of the presented measurements, the jet velocity ratio was set to $u_{j} / u_{1}=1$ for the subsequent measurements in combination with an amplitude modulation frequency of $F=230 \mathrm{~Hz}$. Figures 6 (a) and (b) shows the mass-averaged results for the total pressure loss coefficient $\zeta_{q 1}$, plotted over a half blade span. A comparison of the baseflow losses for both inflow angles shows distinct higher values for the reduced inflow angle. Furthermore, some results with synthetic jet actuation have been added to both figures. It can be seen that the losses are markedly reduced with the applied flow control approach. For $\beta_{1}=55^{\circ}$ at $y /$ $h=-30$ per cent, the position of the corner vortex, improvements of more than 15 per cent can be achieved; whereas at blade midspan a slight loss increase can be observed for some configurations. Comparing the different investigated actuator configurations reveals that the best improvements can be achieved using the sidewall and all blade surfacemounted actuators together. The integral total pressure loss coefficient $\zeta_{t, A F C}$ shows improvements of $\varepsilon=6.8$ per cent compared to the baseflow. For the calculation of $\varepsilon$, the integral total pressure loss, differences are normalized with the total pressure loss for the baseflow using the following formulation

$$
\varepsilon=\frac{\zeta_{t, B a s e}-\zeta_{t, A F C}}{\zeta_{t, \text { Base }}}
$$

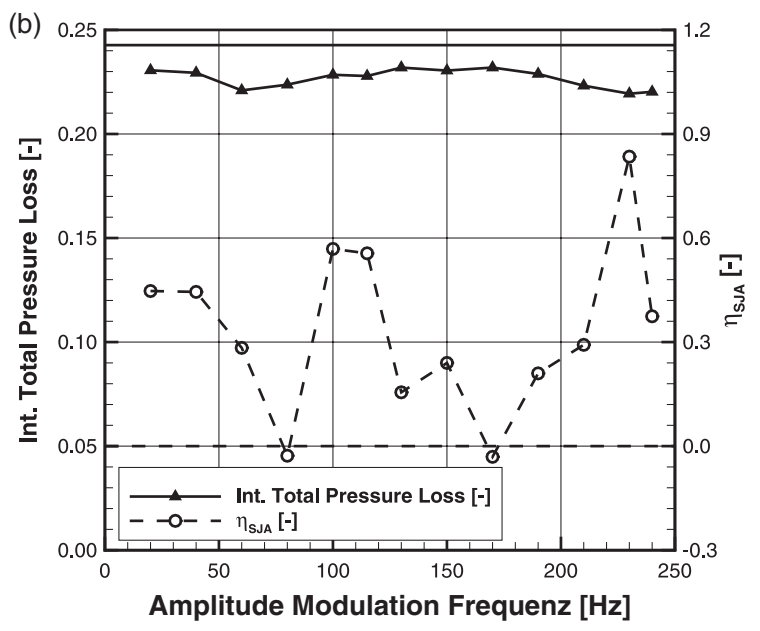

Fig. 5 Losses and efficiency for different SJA parameter 
With only the sidewall actuators activated, a slight improvement in the region of the maximum losses can be achieved. Better results can be obtained in combination with the blade-mounted actuators. Adding the centre actuator seems to be very efficient. A further improvement is possible using the outer blade surface actuators (right/left actuators) in combination with the sidewall actuation. Increasing the number of actuators means to increase the momentum input to the boundary layer and thus results in further improvements.

A slightly different flow behaviour is observed in Fig. 6(b) for $\beta_{1}=60^{\circ}$. A combination of all actuators did not work best for these inflow conditions. Even using the sidewall actuators alone will result in better loss reductions than using all actuators simultaneously. A combination of the sidewall actuators and the blade surface actuator at midspan results in a wake characteristic that is similar to the one of the baseflow. Only the outer blade surface actuators further reduce the losses in the cascade wake in combination with the sidewall-mounted synthetic jets. For this flow control set-up, improvements of 9, 6 per cent can be achieved for the integral total pressure loss.
In contrast to the $\beta_{1}=55^{\circ}$ measurements, remarkable loss reductions can be achieved over the hole blade span. Minimum total pressure losses can be observed for the midspan position, even with a deactivated blade centre actuator. These results show that not only the amount of added momentum is important for this flow control set-up. Even the way it will be added to the flow is crucial. As already observed in Fig. 3, the blade centre actuator is placed downstream of the separation line for an inflow angle $\beta_{1}=60^{\circ}$. The wake measurements show that using an SJA in a poststall configuration results in an increased loss production in the cascade. Thus, remarkable reductions can be achieved using the actuators that are located slightly upstream of the flow separation. The achieved loss reductions $\varepsilon$ for the different actuator configurations are summarized in Table 2.

Figure 7(a) shows on the right half the areal distribution of the total pressure loss differences

$$
\Delta \zeta_{q 1}=\zeta_{q 1, B a s e}-\zeta_{q 1, A F C}
$$

for $\beta_{1}=55^{\circ}$ and on the left the distribution of $\zeta_{q 1, \text { Base }}$. Light colours in the right part of the figure denote regions with reduced losses in case of actuation,
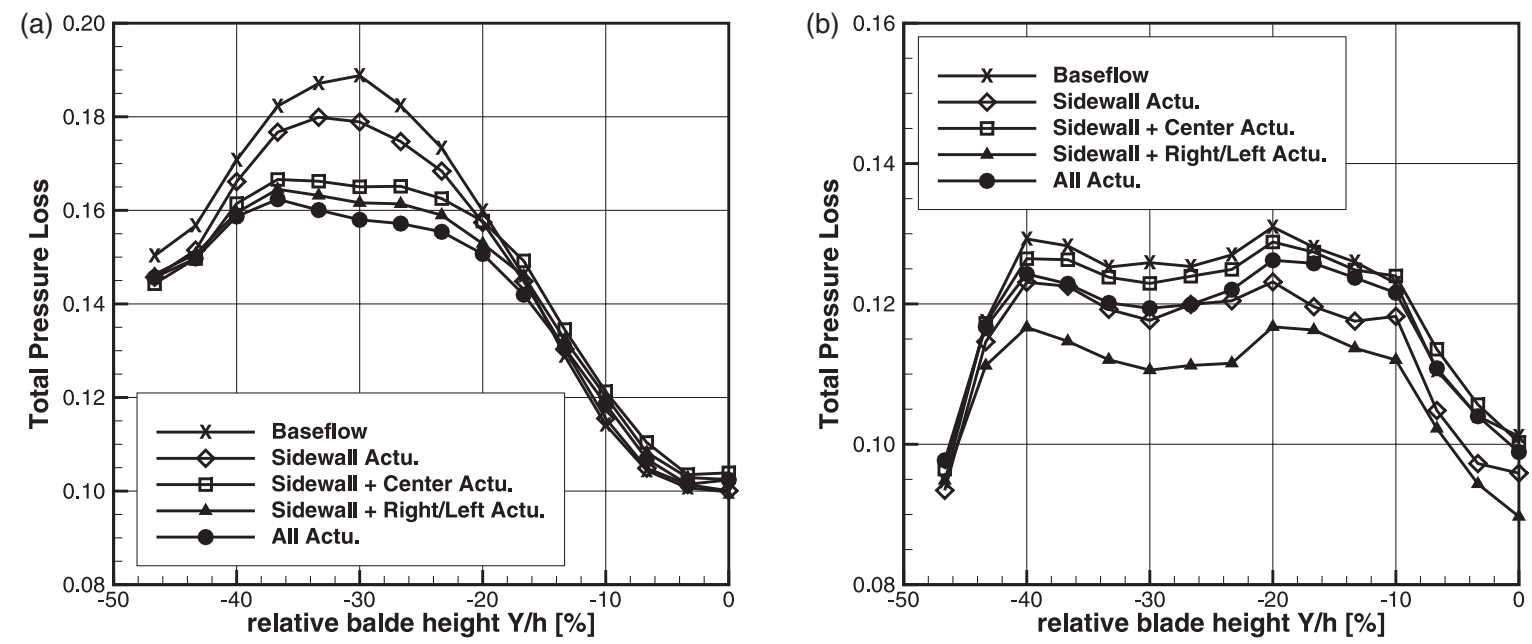

Fig. 6 Wake characteristics with active flow control
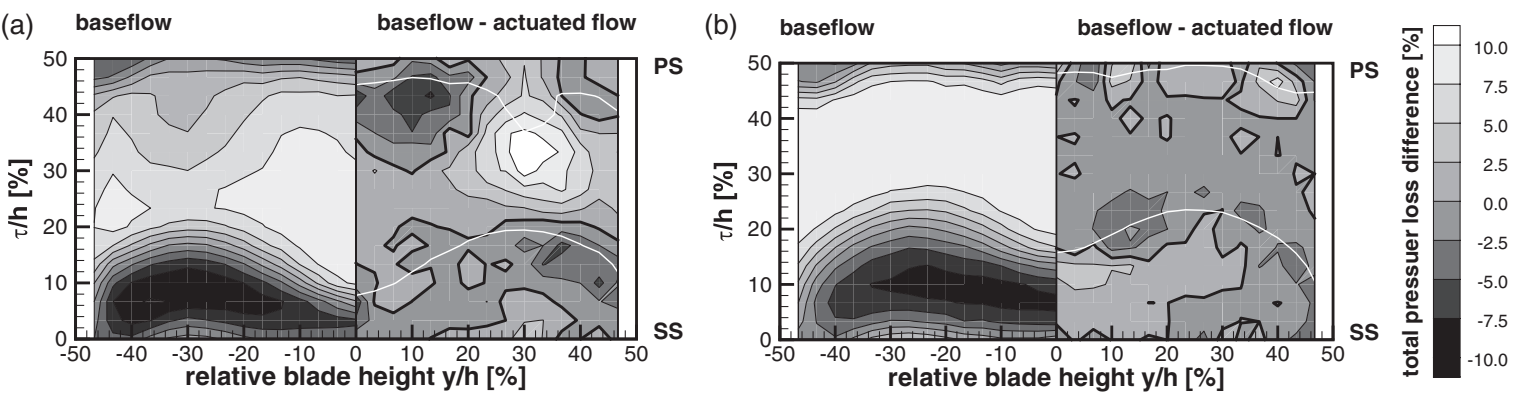

Fig. 7 Total pressure loss with and without flow control 
Table 2 Summary of the SJA experiments

\begin{tabular}{llll}
\hline & Actuator & $\varepsilon[\%]$ & $\eta[\%]$ \\
\hline \multirow{3}{*}{$\beta_{1}=55^{\circ}$} & Sidewall & 2.21 & -0.92 \\
& Sidewall + centre & 3.92 & -0.47 \\
& Sidewall + right/left & 5.35 & -0.10 \\
& All & 6.83 & 0.48 \\
$\beta_{1}=60^{\circ}$ & Sidewall & 5.07 & 0.28 \\
& Sidewall + centre & 0.63 & -0.97 \\
& Sidewall + right/left & 9.61 & 0.83 \\
& All & 2.54 & -0.63 \\
\hline
\end{tabular}

whereas dark areas show increased loss production. The thick black line denotes the zero line. The white line depicts the flow topology of the baseflow configuration to locate the regions with changed losses in the cascade wake. For the comparison, the most effective flow control set-up using all actuators with a frequency of $F=230 \mathrm{~Hz}$ and a jet velocity ratio of $u_{j}$ l $u_{1}=1$ was chosen (Table 2). The contour plot of the total pressure loss differences shows that the pressure losses increase in the area of the suction side vortex depicted by darker contours; whereas strong loss reductions are observed for the second vortex which is located on the pressure side at $y / h=30$ per cent and $\tau / h=35$ per cent. Due to the suction side actuation, these results were not expected. The synthetic jet actuation seems to have a strong upstream effect that influences the LE separation on the pressure side, as can be seen in Fig. 8. The pressure distribution on the suction side remains unchanged whereas the pressure side shows significant changes. The LE separation bubble shortens and causes a faster rise of $c_{p}$ on the blade pressure side. The shape of the $c_{p}$ distribution is now similar to the one for $\beta_{1}=60^{\circ}$ (Fig. 4(a)). The existence of this bubble seems to be responsible for the additional vortex on the pressure side. Nevertheless, further five-hole probe measurements inside the blade passages are needed to fully understand the vortex configuration and the changes in case of AFC for this inflow angle. The LE separation bubble on the pressure side vanishes, increasing the inflow angle to $\beta_{1}=60^{\circ}$. Figure 7 (b) shows the total pressure loss differences for the inflow angle on $\beta_{1}=60^{\circ}$ using the sidewall actuators in combination with the right and left blade surface actuators with $F=230 \mathrm{~Hz}$ and $u_{j} / u_{1}=1$. Distinct loss reductions of up to 5 per cent can be observed in the region of the suction side vortex especially at blade midspan. This is remarkably due to the fact that all actuators except the one at the midspan position were used. The slightly higher losses at $\tau / h=20$ per cent that were displayed along the white line, which marks the position of the suction side vortex, suggest an upwards shift of the corner vortex. This suggestion is confirmed by the reduced pressure losses along the

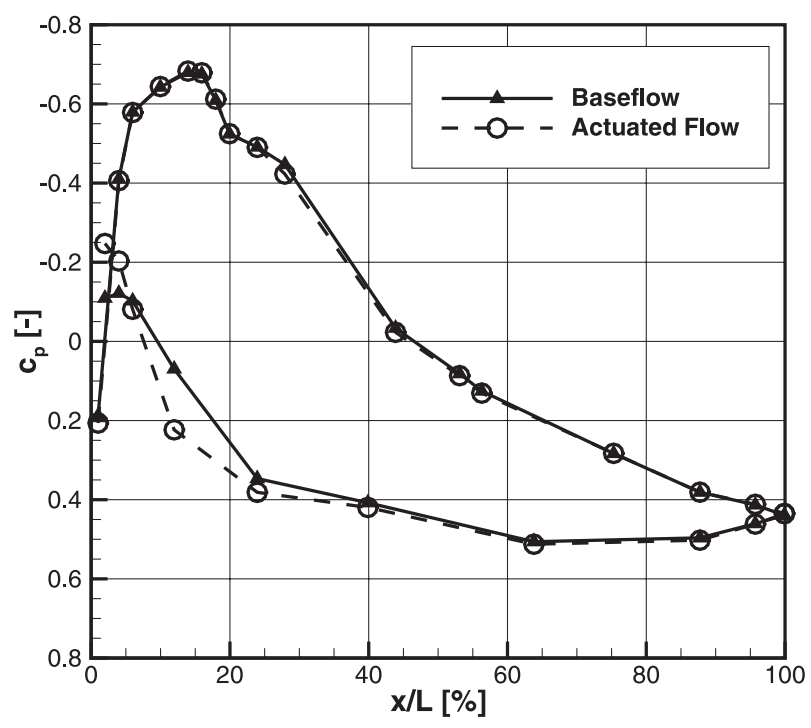

Fig. $8 c_{p}$ for $\beta_{1}=55^{\circ}$ with $\mathrm{AFC}$

vortex line close to the pressure side. For this flow case, the loss reductions are directly related to a reduction of the corner vortices using the SJAs. The right and left blade surface-mounted actuators are placed slightly upstream of the separation line (Fig. 3 ) on the suction side. This shift of the relevant flow phenomena close to the actuator orifices seems to improve the flow control efficiency. The reduction of the total pressure losses does not affect the $c_{p}$ distribution at midspan and is therefore not presented here.

\subsection{Active flow control efficiency}

As reported by other authors like Matejka et al. [8] and Zheng et al. [9], flow control with synthetic jet actuators is highly efficient. Both authors report that the amount of saved work reducing the total pressure loss exceeds the sum of energy spend. Therefore, an efficiency calculation based on the method presented by Matejka et al. [8] has been conducted for the presented measurements as well. The saved up specific energy $\Delta W$ can be calculated from the differences in the total pressure loss between the baseflow and the actuated flow field as follows:

$$
\Delta W=\frac{1}{2}\left(\zeta_{t, 0}-\zeta_{t, A F C}\right) u_{1}^{2}
$$

During the AFC measurements, current and voltage of the synthetic jets have been measured. With the measured values, the actuator power consumption $P_{e l}$ was calculated. Dividing $P_{e l}$ by the passage massflow $\dot{m}$ results in the specific energy spent during actuation

$$
W_{S J A}=\frac{P_{e l}}{\dot{m}}
$$


With these two quantities, the flow control efficiency of the synthetic jet experiments can be calculated. This articl uses a modified definition of the actuator efficiency compared to reference $[\mathbf{8}]$

$$
\eta_{S J A}=\frac{\Delta W-W_{S J A}}{W_{S J A}}
$$

Values above zero for $\eta_{S J A}$ indicate a positive energy balance. This means the energy spent is smaller compared to the saved energy due to the loss reductions in the cascade wake, whereas negative values depict that the energy spent exceeds the gain of the loss reductions. Beside the total pressure loss difference, Table 2 presents also the actuator efficiency for the different actuator configurations. This shows that loss reductions can be achieved for all configurations, but only three configurations show positive values for $\eta_{S J A}$ and therefore a gain for the flow control set-up. The maximum value of $\eta_{S J A}=0.83$ for $\beta_{1}=60^{\circ}$ means gain of more than 80 per cent. Results of the efficiency calculation are also plotted in Figs. 5(a) and (b). In the case of the amplitude variation it can be seen that using jet velocity ratios less than $u_{j} / u_{1}=0.8$ is not efficient. However for higher jet velocities, the amount of saved energy rises faster than the spent energy. For the maximum jet velocity ratio, a maximum efficiency of $\eta_{S J A}=0.89$ is reached for this case. The value of $\Delta W$ is nearly twice as high as the value for the spent energy. The efficiency calculation for the frequency variation plotted in Fig. 5(b) shows a slightly different trend compared to the total pressure loss results. The efficiency maximum is located at $F=230 \mathrm{~Hz}$ which corresponds to the minimum total pressure loss measured. Therefore, a second efficiency peak should be located at $F=60 \mathrm{~Hz}$ due to small loss values. In fact, the second peak is shifted to higher frequencies around $F \approx 110 \mathrm{~Hz}$, which is nearly twice the value of $F=60 \mathrm{~Hz}$ and the half of $F=230 \mathrm{~Hz}$. Higher efficiencies can also be observed for $F<60 \mathrm{~Hz}$ due to the fact that the power consumption of the synthetic jets is reduced with decreasing actuation frequency. The integral total pressure losses for these frequencies are distinctly higher compared to $F=60 \mathrm{~Hz}$. These findings reveal a frequency correlation for higher harmonics of $F \approx 60 \mathrm{~Hz}$. This correlation has to be investigated by further detailed measurements of the cascade flow field. A possible suggestion can be given by the dimensionless frequency $F^{+}$which is close to one for $F=60 \mathrm{~Hz}$. Nevertheless, the calculated efficiencies confirm the findings of other authors that using synthetic jets for flow control applications in turbomachinery is highly efficient.

\section{CONCLUSIONS}

Results of flow control experiments using synthetic jets on a highly loaded compressor cascade to reduce strong secondary flow effects are presented. For that reason, 30 actuators have been mounted to the cascade sidewalls and the blade suction side surface. An individual actuator control enables a wide variation of flow control parameters. Detailed wake measurements with a five-hole probe have been conducted to measure the effects of actuation on the cascade flow field. Based on these results, an efficiency calculation was conducted for the SJAs. This reveals that using SJAs is highly effective for loss reductions on highly loaded compressor blades. Varying the inflow angle of the cascade shows that the SJAs have to be located directly in front of the flow separation to achieve positive effects on the flow field. Furthermore, small injection angles for the actuation perform better than perpendicular jets. An amplitude variation carried out subsequently shows that using high jet velocity ratios of $u_{j} / u_{1}=1$ is most efficient and achieves the best loss reductions. This suggests that flow control is possible in high Mach number flows of real engines, but this has to be demonstrated in further investigations. Varying the actuation frequency depicts the best results for a high frequency of $F=230 \mathrm{~Hz}$. This suggests that using higher harmonic frequencies $F^{+}>1$ of the natural instabilities will result in higher loss reductions and flow control efficiencies. According to the results of the amplitude variation, further experiments have to be realized to account for wake effects that can heavily influence the actuation frequency for flow control applications.

\section{FUNDING}

The presented results were obtained in cooperation with Rolls-Royce Deutschland Ltd. \& Co. KG within the European Union founded research project TATMo-Turbulence and Transition Modeling for Special Turbomachinery Applications, FP6-2005AERO-1-030939.

(C) Authors 2011

\section{REFERENCES}

1 Lord, W. K., MacMartin, D. G., and Tillman, T. G. Flow control opportunities in gas turbine engines. Fluids 2000, Denver 2000, Paper No. AIAA 2000-2234.

2 Prandtl, L. Translation: motion of fluids with very little viscosity, 1928 (Technical Report NACA TM 452, NASA).

3 Liesner, K., Meyer, R., Lemke, M., Gmelin, C., and Thiele, F. On the efficiency of secondary flow suction 
in a compressor cascade. In Proceedings of ASME Turbo Expo 2010: Power for land, sea and air, Glasgow, UK, 2010, Paper No. GT2010-22336.

4 Nerger, D., Saathoff, H., Radespiel, R., Gümmer, V., and Clemen, C. Experimental investigation of endwall and suction side blowing in a highly loaded compressor stator cascade. In Proceedings of ASME Turbo Expo 2010: Power for land, sea and air, Glasgow, UK, 2010, Paper No. GT2010-22578.

5 Seifert, A., Bachar, T., Koss, D., Shepshelovich, M., and Wygnanski, I. J. Oscillatory blowing: A tool to delay boundary-layer separation. AIAA J., 1993, 31, 2052-2060.

6 Hecklau, M., Zander, V., Peltzer, I., Nitsche, W., Huppertz, A., and Swoboda, M. Experimental AFC approaches on a highly loaded compressor cascade. In Active flow control II - NNFM 108 (Ed. R. King), 2010, pp. 171-186 (Springer) Notes on Numerical Fluid Mechanics and Multidisciplinary Design.

7 Smith, B. L. and Glezer, A. Vectoring and small-scale motions effected in free shear flows using synthetic jet actuators. In Proceedings of the 35th AIAA Aerospace sciences meeting and exhibit 1997, Paper No. AIAA 97-0213.

8 Matejka, M., Safarik, P., Popelka, L., and Nozicka, J. Influence of active methods of flow control on compressor blade cascade flow. ASME Turbo Expo 2008, Paper No. GT2008-51109.

9 Zheng, X., Zhou, S., Lu, Y., Hou, A., and Li, Q. Flow control of annular compressor cascade by synthetic jets. J. Turbomach., 2008, 130(021018), 1-7.

10 Glezer, A. and Amitay, M. Synthetic jets. Annu. Rev. Fluid Mech., 2002, 34, 503-529.

11 Gmelin, C., Steger, M., Thiele, A., F., Huppertz, and Swoboda, M. Unsteady rans simulations of a highly loaded low aspect ratio compressor stator cascade with active flow control. In Proceedings of ASME Turbo Expo 2010: Power for land, sea and air, Glasgow, UK, 2010, Paper No. GT2010-22516.

12 Zander, V., Hecklau, M., Nitsche, W., Huppertz, A., and Swoboda, M. Active control of corner vortices on a highly loaded compressor cascade. In Proceedings of the 8th European Turbomachinery Conference, 2009 (Graz).

\begin{tabular}{|c|c|}
\hline$h$ & blade height $(\mathrm{mm})$ \\
\hline$L$ & chord length $(\mathrm{mm})$ \\
\hline$M a$ & Mach number $\quad(-)$ \\
\hline$p$ & pressure (Pa) \\
\hline$P_{e l}$ & electric power (W) \\
\hline$q$ & dynamic pressure $(\mathrm{Pa})$ \\
\hline $\operatorname{Re}$ & Reynolds number $\quad(-)$ \\
\hline$s$ & length from the leading edge $(\mathrm{mm})$ \\
\hline$S$ & total length of blade side (mm) \\
\hline$T u$ & turbulence intensity $(-)$ \\
\hline$u$ & velocity $(\mathrm{m} / \mathrm{s})$ \\
\hline$w$ & slot width (mm) \\
\hline$W$ & specific energy $\left(\mathrm{m}^{2} / \mathrm{s}^{2}\right)$ \\
\hline$x, y$ & coordinates (mm) \\
\hline$\beta$ & flow angle $\quad\left(^{\circ}\right)$ \\
\hline$\Delta \beta$ & turning $\quad\left(^{\circ}\right)$ \\
\hline$\gamma$ & stagger angle $\left.\quad{ }^{\circ}\right)$ \\
\hline$\varepsilon$ & pressure loss differences $(-)$ \\
\hline$\zeta_{q 1}$ & pressure loss coefficient $\quad(-)$ \\
\hline$\rho$ & density $\quad\left(\mathrm{kg} / \mathrm{m}^{3}\right)$ \\
\hline$\eta$ & actuator efficiency $\quad(-)$ \\
\hline$\tau$ & pitch (mm) \\
\hline$\varphi$ & injection angle $\quad\left(^{\circ}\right)$ \\
\hline
\end{tabular}

Subscripts

0 baseflow

1 inlet

$2 \quad$ outlet

s static

t total

j jet

Abbreviations

AFC active flow control

LE leading edge

SJA synthetic jet actuator

TE trailing edge

\section{APPENDIX}

\section{Notation}

$\begin{array}{lll}c_{p} & \text { pressure coefficient }(-) \\ d & \text { slot depth } & (\mathrm{mm}) \\ F & \text { frequency } & (1 / \mathrm{s})\end{array}$

